\title{
In Vivo MRI Assessment of Knee Cartilage in the Medial Meniscal Tear Model of Osteoarthritis in Rats
}

\author{
Zhiyong Xie ${ }^{1}$, Serguei Liachenko ${ }^{2, *}$, Ping-Chun Chiao ${ }^{1}$, Santos Carvajal-Gonzalez ${ }^{1}$, \\ Susan Bove ${ }^{1}$, and Thomas Bocan ${ }^{1}$ \\ ${ }^{1}$ Pfizer Inc, Groton, CT, United States \\ \{zhiyong.Xie, Ping-Chun.Chiao\}@pfizer.com, \\ \{Santos.Carvajal-Gonzalez, Susan.Bove, Thomas.Bocan\} @pfizer.com \\ ${ }^{2}$ NCTR/FDA, Jefferson, AR, United States \\ serguei.liachenko@fda.hhs.gov
}

\begin{abstract}
We present a new approach for quantifying the degradation of knee cartilage in the medial meniscal tear (MMT) model of osteoarthritis in the rat. A statistical strategy was used to guide the selection of a region of interest (ROI) from the images obtained from a pilot study. We hypothesize that this strategy can be used to localize a region of cartilage most vulnerable to MMT-induced damage. In order to test this hypothesis, a longitudinal study was conducted in which knee cartilage thickness in a pre-selected ROI was monitored for three weeks and comparisons were made between MMT and control rats. We observed a significant decrease in cartilage thickness in MMT rats and a significant increase in cartilage thickness in sham-operated rats as early as one week post surgery when compared to pre-surgery measurements.
\end{abstract}

Keywords: Osteoarthritis, Preclinical MRI, Cartilage segmentation, Surface registration, Thickness statistics.

\section{Introduction}

One of the major hallmarks of osteoarthritis (OA) is degradation of the articular cartilage in weight bearing joints [1]. The morphology of the articular cartilage is considered to be the primary marker of OA progression. Magnetic resonance imaging (MRI) has been used to assess the anatomical/functional integrity of the knee structure and has shown great potentials in clinical studies [2][3][4][5][6]. However, in preclinical research where small animals are widely used to study the pathogenesis of OA and evaluate efficacy of drugs [7], the use of MRI has been limited due to the challenges of small animal in-vivo imaging and image analysis. Because of mediocre image contrast and thin cartilage structure relative to the image resolution, the full extent of the cartilage surface and its spatial morphological variation cannot be accurately determined in MR images of knee joints in small animal preclinical imaging without advanced image manipulation.

This paper presents a MR imaging and data analysis method to assess knee cartilage in the rat medial meniscal tear (MMT) model. The MMT model is a surgically-induced

* All of the work described in this paper was performed while Serguei Liachenko was employed by Pfizer Inc. Nothing should be interpreted as an opinion of NCTR/FDA. 
model of OA that results in rapid degradation of cartilage [7]. Results from a previous in-house MRI study indicated that the MMT model induced cartilage degeneration in a similar region on the medial tibia for all rats. However, the degeneration was not noticeable if we use the average thickness of whole cartilage as a metric. To maximize the sensitivity in detecting and quantifying MMT-induced morphological changes in articular cartilage, we propose a statistical strategy to guide the selection of a region of interest (ROI) on the medial tibia and hypothesize that this strategy can localize an area of cartilage most vulnerable to MMT-induced damage. To test this hypothesis, we performed a pilot study to identify the ROI based on the MR images of five MMT and six sham-operated rats. The same ROI was then applied to the images collected in a longitudinal study in which MR images of two groups of rats (MMT vs. sham) were scanned before surgery, and again one and three weeks after surgery. In order to determine repeatability, every rat was re-scanned four times in one day. Average thickness of the cartilage inside the predefined ROI was measured blindly for each image. Results show that the proposed method can detect MMT-induced cartilage degeneration as early as one week post surgery.

\section{Materials and Methods}

\subsection{Animals and Experimental Design}

All animal handling procedures were carried out in compliance with the NIH Guide for the Care and Use of Laboratory Animals under a protocol approved by the Pfizer Global Research and Development Animal Care and Use Committee.

The first part of this experiment was a pilot study which was used to identify the regions of the cartilage that were most vulnerable to MMT-induced damage. Eleven male Sprague-Dawley rats $(415 \pm 22 \mathrm{~g})$ were used in the pilot study. Five rats were randomly selected to receive MMT surgery and the other six rats served as a control (sham-operated) group. OA was surgically induced by transection of the medial collateral ligament and medial meniscus of the femoro-tibial joint as described previously [8]. To reduce the bias caused by the pain of the surgery, rats in control group received sham preparation in which the medial collateral ligament was exposed, but not transected. Knee joint MR images were acquired three weeks after the surgery. A ROI was determined based on the thickness statistics of the medial tibial cartilage in these images.

In the longitudinal scan-rescan study, eight male Sprague Dawley rats $($ MMT N = 4 , and sham $\mathrm{N}=4$ ) underwent MMT or sham surgery. MRI was performed prior to surgery and then again one and three weeks post surgery. In order to determine repeatability of the image acquisition, each animal was re-scanned four times during a single imaging session (one day). Rats were taken out of the magnet and removed from the cradle between the scans, their legs were massaged for 2 minutes and they were repositioned back into the cradle and MRI scanner. The average thickness of the cartilage inside the predefined ROI was computed from the acquired images.

\subsection{Image Acquisition}

Knee joint MRI was performed on a 7T Bruker Biospec scanner equipped with $12 \mathrm{~cm}$ ID gradient insert (up to $20 \mathrm{G} / \mathrm{cm}$ ). Animals were anesthetized using isoflurane 
(3\% induction with $1.2-1.5 \%$ maintenance) in oxygen. Rats were placed in a supine position on heated cradle, their right knee was flexed to an angle of $105^{\circ}$ and secured to the animal holder to prevent motion. An actively decoupled, curved quadrature receive-only surface coil (mouse brain coil, Bruker BioSpin) was placed on the knee and the cradle was then placed inside the magnet. RF excitation was delivered using a $72 \mathrm{~mm}$ ID birdcage volume resonator. An i.v. bolus of Magnevist巴 $(0.4 \mathrm{ml} / \mathrm{kg})$, followed by a constant infusion $(0.44 \mathrm{ml} / \mathrm{kg} / \mathrm{hr})$ was delivered to improve synovial fluidcartilage delineation. Optimal slice planning was performed using information obtained from quick orthogonal images in the coronal, axial and sagittal planes. High resolution 3D SPGR anatomical images were acquired in the sagittal plane with the following acquisition parameters: $\mathrm{TE}=3.6 \mathrm{~ms}, \mathrm{TR}=25 \mathrm{~ms}, \mathrm{FA}=30^{\circ}, \mathrm{NA}=6$, MTX $=512 \times 170 \times 64$, resolution of $29 \times 116 \times 231 \mu \mathrm{m}$. The left pane of figure 1 shows the representative MR image of the knee joint of a rat.

\subsection{Image Analyses}

The tibia and tibial cartilage in collected images were segmented automatically using internally developed tools (see below). Segmentation results were reviewed and errors were manually corrected by a trained expert who was blinded to the treatment group. After the segmentation, a cartilage thickness map on the tibial surface from each image was created and mapped to a template tibial surface. A statistical analysis was performed on aligned cartilage thickness maps to detect the regions where the cartilage thickness was significantly different between the MMT and control groups.

The tibia was segmented for two purposes: first, it provided a spatial reference for the location of the cartilage which helped the segmentation of tibial cartilage; second, it was used to align cartilages from different images to identify the regions which were most vulnerable to MMT-induced damage.

Tibia Segmentation. A surface-to-image registration method was used to segment the tibia on the MR image [9]. Briefly, we selected one MR knee joint image as a template and delineated the tibia in the image using a semi-automatic tool [10]. The segmentation was converted to a surface representation of the tibia. The template tibia surface was fitted to other MR images with weighted external force constraints in which weights and forces were determined by gradients and local intensity profiles obtained from images. To accomplish this fitting, edge points obtained from each MR image set were searched along normal directions of the template tibia surface and the local intensity profiles at these points were extracted and compared to those obtained from the template image. Correlation between intensity profiles was used to select the best-matched edge point. Transformations were then computed to minimize the sum of weighted square distance between transformed atlas surface points and their corresponding best-matched edge points; the weights were again determined by the level of correlation between intensity profiles. To stabilize this fitting process, a sequence of transformations consisting of translation, rigid, affine, and multi-level B-splines, was employed to iteratively morph the bone surface of atlas into the individual tibial structure in the MR image. An example of the segmentation result is presented in the right pane of figure 1 . 
Cartilage segmentation. Various methods have been proposed for cartilage segmentation [11][12]. Our method segments medial tibial cartilage in three sequential steps: cartilage edge identification, tibia evolution, and cartilage labeling. The first step identifies edge pixels of the cartilage from all edge pixels detected by the Canny-Edge algorithm in the whole image. The edge identification is guided by the prior information about the intensity pattern of the cartilage edge and its location relative to the tibia surface. The model is similar to the one described by Kapur et al. [13]. In the second step, the identified edge pixels attract a curve to the cartilage boundary based on a deformable model. Since the tibial cartilage is a thin tissue connected to the tibia, we expand the tibia boundary to the identified cartilage edge pixels rather than evolving a seed inside the cartilage. The final step labels the new included region of the tibia as tibial cartilage. An example of the segmentation result is presented in the right pane of figure 1 .
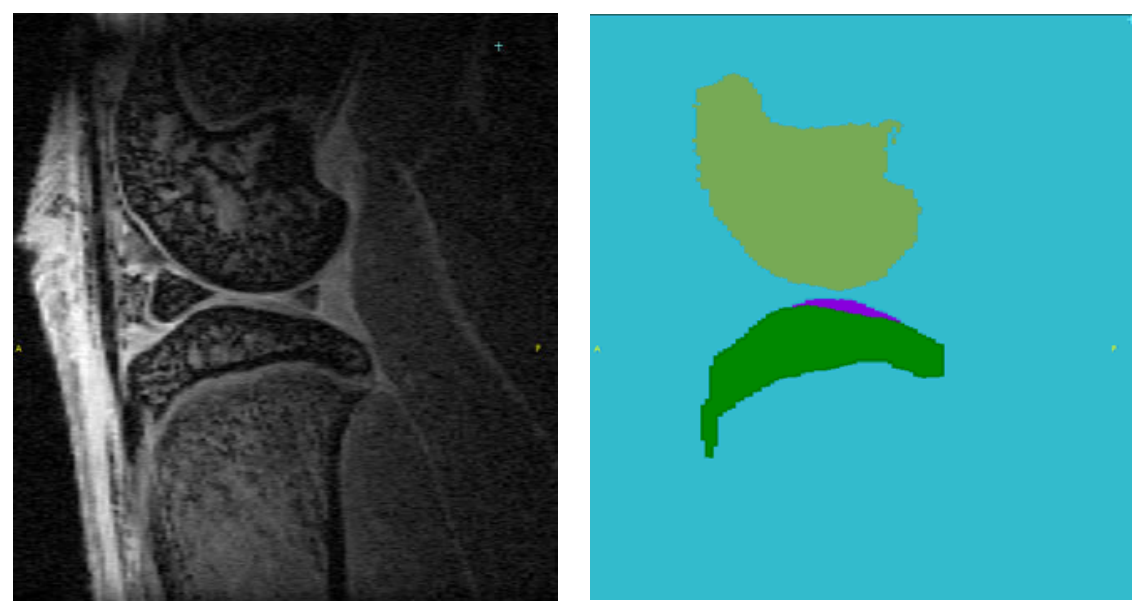

Fig. 1. MRI image of the knee joint (left) of a rat and its segmentation of tibia (green), femur (yellow), and tibial cartilage (magenta, right)

ROI determination. The region of cartilage most vulnerable to MMT-induced damage was identified using statistical parametric mapping based on the images from 11 rats (MMT $\mathrm{N}=5$, and sham $\mathrm{N}=6$ ) in the pilot study. After cartilage segmentation, a cartilage thickness map was computed by sampling cartilage thickness estimates on each vertex of the tibial surface. One example of a cartilage thickness map is shown in the left pane of figure 2. All cartilage thickness maps from 11 subjects can be aligned to a template by registering each individual tibial surface to the template tibial surface using the method described by Xie and Farin [14]. Once the thickness map is created and mapped to the template tibial surface, statistical analysis can be performed at each vertex of the tibial surface across the subjects in the same way as proposed by Lerch and Evans or by Worsley et al $[15,16]$. The right pane of figure 2 illustrates the procedure of this thickness statistical analysis. 

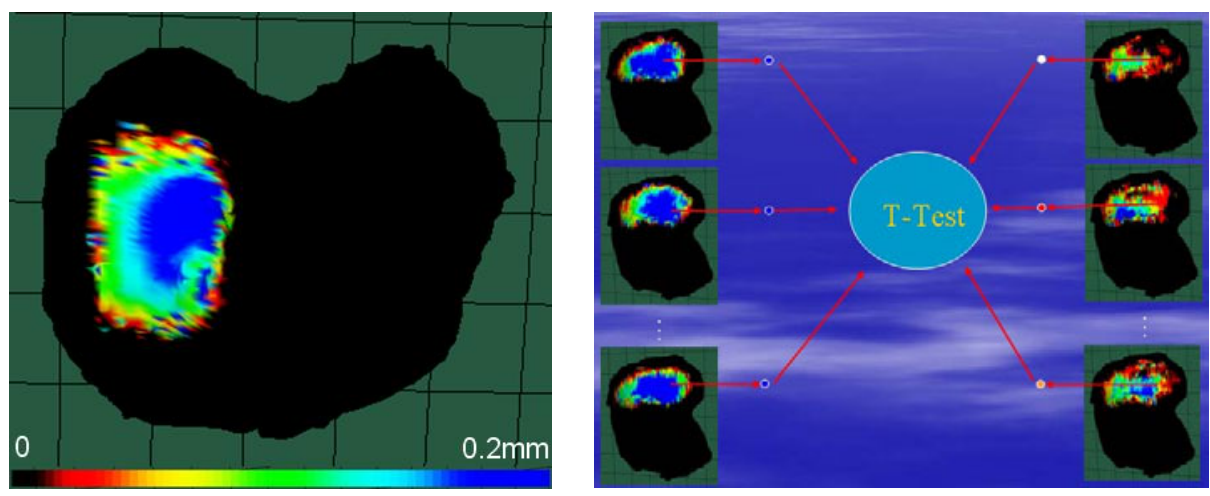

Fig. 2. Cartilage thickness map on the tibial surface (left) and thickness statistical analysis (right). After aligning all cartilage thickness maps to the same template, cartilage thickness at the same vertex can be compared across the subjects to determine group differences.

Using the thickness statistics, we created a p-value map which shows the significance of the thickness difference between two groups at each vertex. The left picture of figure 3 shows the p-value map on the template tibial surface. The dark region has a p-value less than 0.001, which indicates that the cartilage thickness in these regions is significantly different between the two groups. The dark region in the middle of the cartilage was selected and the ROIs were programmatically delineated for the subsequent analysis of the regional cartilage thickness. The right pane of figure 3 shows the ROI defined on the tibial surface.
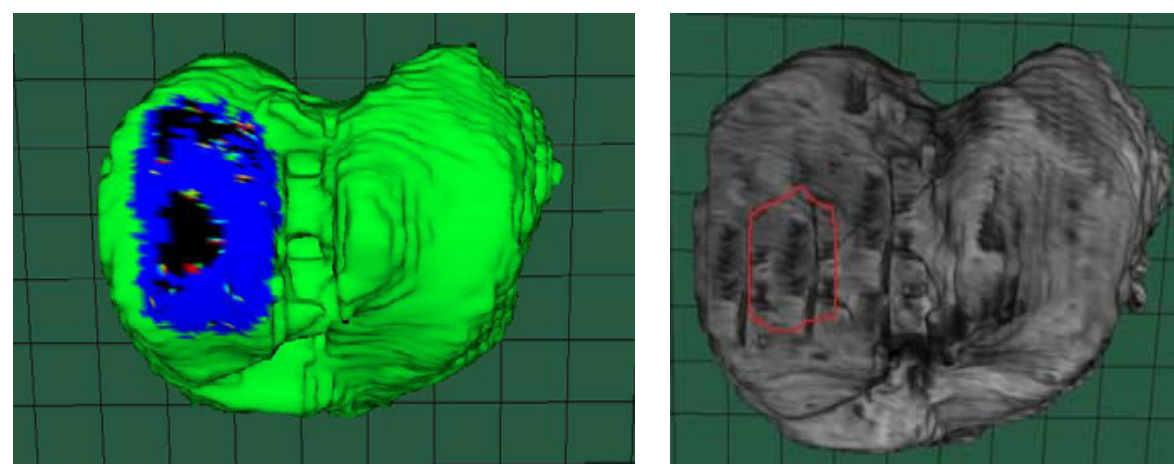

Fig. 3. Left, p-value map after thickness statistical analysis between MMT and sham groups. Right, defined ROI (region enclosed by the red line) based on thickness statistical analysis.

\section{Results}

After images in the longitudinal study were collected and segmented, a thickness map was created for each image. The ROI defined from the pilot study was transformed to the thickness map by registering the template tibial surface to the tibial surface of the 
subject. The average thickness of the cartilage inside the ROI was computed for every image.

To compare each group, the ANOVA model included terms for Scan, Weeks, Group as fixed effects, and Scan $\times$ Weeks $\times$ Group as an interaction term. In order to consider the repeated measure, an animal within the scan was introduced as a random effect. Figure 4 shows the least squares mean of cartilage thickness and standard error for each group at each nominal time point (i.e. Weeks 0, 1 and 3).

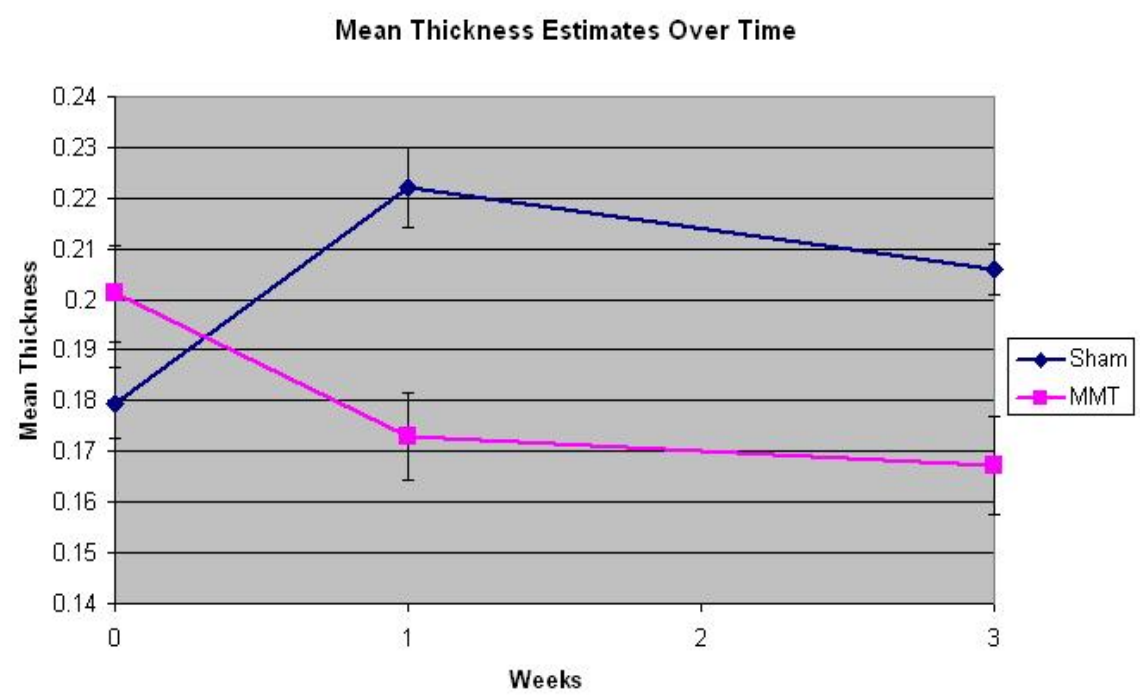

Fig. 4. Least squares means estimates for mean thickness over time and for each group of rats

Based on the statistical analysis, the MMT group had significantly thinner cartilage compared to the sham group at weeks 1 and $3(\mathrm{p}=0.0030$ and $\mathrm{p}=0.0181$, respectively). Within the MMT group, the cartilage thickness was significantly decreased at weeks 1 and 3 when compared to the pre-surgery scan at week $0(p=0.0238$ and $p=$ 0.0073 , respectively). For the sham group, the cartilage thickness was significantly increased at weeks 1 and 3 compared to week $0(p=0.0238$ and $p=0.0073$, respectively).

The coefficient of variation $(\mathrm{CV})$ of the mean thickness was computed for each rat. For the MMT group the CV varied between $4.87 \%$ and $27.53 \%$. For the sham group, the CV was in the range between $4.45 \%$ to $20.62 \%$.

To verify that MMT induced damage was located on a similar region as we detected in the pilot study, we performed thickness statistics for the data collected at weeks 1 and 3. Figure 5 shows the p-value map between the MMT and sham groups at week 1 (left) and week 3 (right). We can see the dark region (with p-value less than 0.001 ) located in a similar region to the one from the pilot study (left pane of the figure 3). 

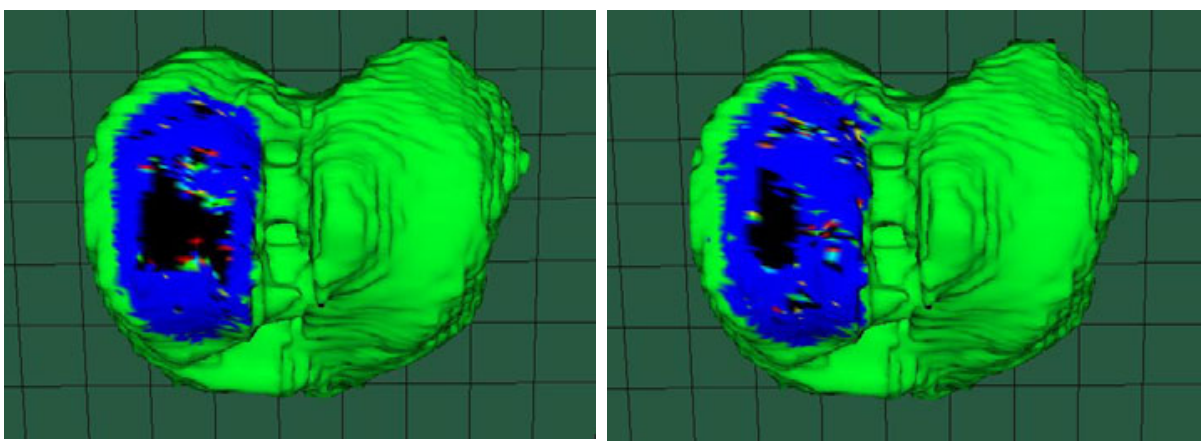

Fig. 5. P-value map generated by statistical comparison between MMT and Sham controls 1 week (left) and three weeks (right) post surgery

\section{Discussion}

In this study we demonstrated that MMT surgery induces a consistently located cartilage lesion on the medial tibial plateau of the rat knee joint. We have proposed a method to identify this region based on MR images from a pilot study. The identified region was then used as the region of interest to monitor MMT-induced cartilage degradation in a longitudinal study. Results of the longitudinal study showed for the first time that the proposed method could detect a significant decrease in cartilage thickness as early as one week post surgery, which indicates the increased sensitivity of the proposed approach. Surprisingly, the sham-operated group showed the increase in the cartilage thickness, which may be attributed to several factors. First, the animals used for this study were still growing, and sham data may reflect just this. Second, the surgery by itself may serve as a stimulator of cartilage growth, which means that similar studies should always be performed with sham operated animals as a control group. In our study we showed that the use of sham operation increased the power of statistical analysis as seen in figure 4 .

The biggest concern of this study is the repeatability. In the worst case, the CV of the mean thickness of 4 repeat scans was $27.53 \%$. We believe this is due to limited image resolution and contrast to noise ratio. Our in-plane resolution is $29 \times 116 \mu \mathrm{m}$. Considering the average cartilage thickness is $200 \mu \mathrm{m}$, one pixel in the image will account for at least $15 \%$ of the cartilage thickness. The situation is even worse if we take into account the big slice thickness which is $231 \mu \mathrm{m}$ ). A slight difference in imaging position and orientation may cause a significant variation in the final measurement. Higher image resolution is highly desired to improve the repeatability and sensitivity for evaluation of potential protective effects of disease modifying compounds for osteoarthritis in the MMT model.

Theoretically, this approach could be translated to human studies, which will also benefit from the higher resolution (due to larger objects and wide availability of fast parallel imaging techniques) and better contrast in clinical MRI. 


\section{References}

1. Poole, A.R.: An introduction to the pathophysiology of osteoarthritis. Frontiers in bioscience 15(4), 662-670 (1999)

2. Eckstein, F., Reiser, M., Englmeier, K., Putz, R.: In vivo morphometry and functional analysis of human articular cartilage with quantitative magnetic resonance imaging - from image to data, from data to theory. Anatomy and Embryology 203(3), 147-173 (2001)

3. Gold, G.E., Beaulieu, C.F.: Future of MR imaging of articular cartilage. Semin Musculoskelet Radiol. 5(4), 313-327 (2001)

4. Recht, M.P., Goodwin, D.W., Winalski, C.S., White, L.M.: MRI of Articular Cartilage: Revisiting Current Status and Future Directions. Am. J. Roentgenol. 185(4), 899-914 (2005)

5. Lang, P., Noorbakhsh, F., Yoshioka, H.: MR Imaging of Articular Cartilage: Current State and Recent Developments. Radiol. Clin. N Am. 43, 629-639 (2005)

6. Peterfy, C., Gold, G., Eckstein, F., Cicuttini, F., Dardzinski, B., Stevens, R.: MRI protocols for whole-organ assessment of the knee in osteoarthritis. Osteoarthritis and Cartilage 14, 95-111 (2006)

7. Bendele, A.M.: Animal models of osteoarthritis. J. Musculoskelet Neuronal Interact. 1(4), 363-376 (2001)

8. Bove, S., Laemont, K., Brooker, R., Osborn, M., Sanchez, B., Guzman, R., Hook, K., Juneau, P., Connor, J., Kilgore, K.: Surgically induced osteoarthritis in the rat results in the development of both osteoarthritis-like joint pain and secondary hyperalgesia. Osteoarthritis and Cartilage 14(10), 1041-1048 (2006)

9. Xie, Z., Tamez-Pena, J., Liachenko, S., Dhamija, S., Gieseg, M., Chiao, P.: Segmentation by surface-to-image registration. In: Proceedings of SPIE Medical Imaging 2006 (2006)

10. Tamez-Pena, J., Parker, K.J., Totterman, S.: Unsupervised statistical segmentation of multispectral volumetric MR images. In: Proceedings of SPIE Medical Imaging 1999 (1999)

11. Solloway, S., Taylor, C.J., Hutchinson, C.E., Waterton, J.C.: Quantification of Articular Cartilage from MR Images Using Active Shape Models. In: Buxton, B.F., Cipolla, R. (eds.) ECCV 1996, Part II. LNCS, vol. 1064, pp. 400-411. Springer, Heidelberg (1996)

12. Fripp, J., Crozier, S., Warfield, S.K., Ourselin, S.: Automatic Segmentation of Articular Cartilage in Magnetic Resonance Images of the Knee. In: 10th International Conference on Medical Image Computing and Computer Assisted Intervention, Brisbane, Australia, pp. 186-194 (2007)

13. Kapur, T., Beardsley, P., Gibson, S., Grimson, W., Wells, W.: Model-based segmentation of clinical knee MRI. In: Proc. IEEE Int'l. Workshop on Model-Based 3D Image Analysis, pp. 97-106 (1998)

14. Xie, Z., Farin, G.E.: Image Registration Using Hierarchical B-Splines. IEEE Transactions on Visualization and Computer Graphics 10(1), 85-94 (2004)

15. Lerch, J.P., Evans, A.C.: Cortical thickness analysis examined through power analysis and a population simulation. NeuroImage 24(1), 163-173 (2005)

16. Worsley, K.J., Taylor, J.E., Carbonell, F., Chung, M.K., Duerden, E., Bernhardt, B., Lyttelton, O., Boucher, M., Evans, A.C.: SurfStat: A Matlab toolbox for the statistical analysis of univariate and multivariate surface and volumetric data using linear mixed effects models and random field theory. NeuroImage, OHBM poster (2009) (accepted) 